\title{
Suppression of Stress and Crack Generation in Local Glass Melting by Picosecond Laser Irradiation at a High Repetition Rates with Temporal Energy Modulation
}

\author{
Akinao Nakamura $^{1}$, Masaaki Sakakura ${ }^{1,2}$, Yasuhiko Shimotsuma ${ }^{1}$, Kiyotaka Miura ${ }^{1}$ \\ ${ }^{1}$ Department of Material Chemistry, Graduate School of Engineering, \\ Kyoto University, Kyoto 615-8510, Japan; \\ E-mail: miura2@ func.mc.kyoto-u.ac.jp \\ ${ }^{2}$ Next Generation Laser Processing Technology Research Association, Kyoto 615-8245, Japan
}

\begin{abstract}
Modification lines by local melting inside glass were written by focusing picosecond laser pulses at high repetition rate with temporal modulation of pulse energies. It was found that straight modification lines with homogeneous stress distribution was able to be written by ps laser irradiation with temporal energy modulation, while modification lines written by ps laser irradiation without modulation had curved boundaries and concentrated stress. The threshold of the crack generation was higher with temporal energy modulation $(22 \mu \mathrm{J}$ of the average pulse energy) than that without modulation (16.5 $\mu \mathrm{J}$ of the average pulse energy), which resulted in about 1.3 times larger width of modification line with modulation than that without modulation. In addition, the elongation of a modification line in the laser propagation direction could be suppressed with modulation. The mechanism of the suppression of stress and crack generation with temporal energy modulation will be discussed according to the movement of light absorbed region during laser irradiation.
\end{abstract}

DOI: 10.2961/jlmn.2017.02.0014

Keywords: ultrafast laser, glass, welding, temporal modulation, processing

\section{Introduction}

High repetition rate ultrashort pulsed lasers have been attracting much interest as a tool for local melting [1-4] and micro-welding of transparent materials such as glasses [5-8]. Local melting occurs by nonlinear photoexcitation [9] and heat accumulation around the photoexcited region [1-4]. For laser welding, the local melting is induced at the interface between two glasses to join them as shown in Figure 1 (a) [4-7]. Because the molten region is confined by solid materials, thermal damages that often occur on the free surface of solids can be minimized [4]. Welding by ultrashort pulsed laser has an advantage because glass plates can be joined without adhesives or glass frits [10].

Local melting by laser irradiation at a high repetition rate depends on the pulse duration. The modification line written inside alkali-silicate glass plates by 100 fs laser pulses and that by 10 ps laser pulses are shown in Fig. 1 (b) and 1 (c), respectively. Both the modification lines by fs laser and ps laser have two structures; one is the central region, in which glass has been molten during laser irradiation, and the other is the outer region, which has been formed mainly by temperature elevation due to thermal diffusion [1-3,11]. However, the smoothness of the modification lines is completely different. While the modification line by fs laser irradiation is smooth, that by $10 \mathrm{ps}$ laser irradiation has irregular structures in the central region and randomly curved boundaries in the outer region. From the difference, fs laser is much better for glass welding with high stability. However, ps lasers are more attractive for industrial applications, because they are compact and obtained with lower prices. Therefore, finding a way to write smooth modification lines inside glasses is important for application of ps lasers to microwelding of
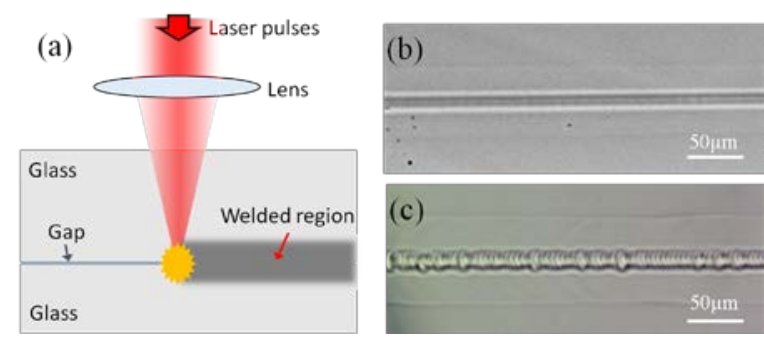

Fig. 1 (a) Schematic illustration of welding of glasses by ultrashort pulsed laser irradiation. (b), (c) Transmission optical microscope images of modification lines written inside an alkali silicate glass by 100 fs laser pulses and 10 ps laser pulses, respectively. 
glasses.

The dependence on the pulse duration can be under stood by two mechanism of photoexcitation, nonlinear and linear absorption [9,11-13]. Nonlinear photoexcitation is dominant by fs laser irradiation, while linear absorption by excited electrons increases as the pulse duration becomes longer because of longer interaction time. Recently, numerical calculation by Miyamoto et al [14] showed that linear absorption of ps laser pulse by thermally excited electrons becomes dominant during local melting of a glass by ps laser irradiation at a high repetition rate. Also, their research groups have observed the periodic movement of highly absorptive plasma in the laser propagation direction by ultrafast imaging $[14,15]$ and attributed the periodic patterns in a modification line to the periodic movement of plasma. Their observation showed that the absorbed region moves suddenly in laser propagation direction during the periodic movement. It is possible that this sudden movement can induce a sudden cooling in the molten region, which can generate a large stress in the molten region. Based on their findings, we speculated that the sudden movement of absorbed region could be responsible for the formation of irregular structures by ps laser irradiation. Therefore, the suppression of the sudden movement should be a key to write homogeneous modification lines inside glasses by ps laser.

In this paper, we proposed a method to suppress the stress generation during writing of molten modification lines inside a glass by ps laser irradiation. In this method, the energies of ps laser pulses were modulated temporally at several $\mathrm{kHz}$. We found that the stress concentration can be suppressed and the threshold of crack generation becomes higher with temporal energy modulation. In addition, we investigated the effect of the pattern (or waveform) of the modulation on the modification line writing. Based on the comparison between modification lines written under different modulations, we discussed the

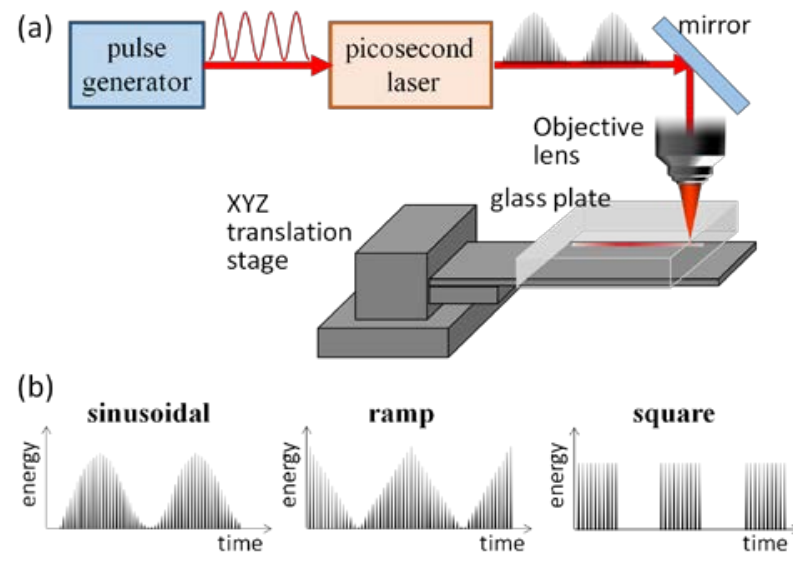

Fig. 2 (a) Laser irradiation setup for writing modification lines inside a glass plate by irradiation with focused picosecond laser pulses. (b) Three patterns of temporal energy modulation.

Table 1 Specification of laser

\begin{tabular}{lc}
\hline Repetition rate & $<1 \mathrm{MHz}$ \\
Average power & $<60 \mathrm{~W}$ \\
Pulse duration & $\sim 10 \mathrm{ps}$ \\
Wavelength & $1064 \mathrm{~nm}$ \\
\hline
\end{tabular}

mechanism of the suppression of stress generation.

\section{Method}

\subsection{Laser irradiation setup}

Figure 2 shows schematic illustration of laser irradiation setup for local melting inside glass plates. A picosecond pulsed laser (Diode pumped $\mathrm{Nd}: \mathrm{YVO}_{4}$ laser, EKSPLA Atlantic) of $1064 \mathrm{~nm}$ in wavelength and $10 \mathrm{ps}$ in pulse duration was used as a laser source. The specification is shown in Table 1 . The laser pulses were focused inside a glass plate placed on a XYZ translation stage (ALIO Industries; AI-LM-10000-XY\& AI-VC-2400-Z-CB) with a $20 \times$ objective lens of numerical aperture of 0.45 (Nikon LU Plan). During laser irradiation, the glass plate was translated normal to the laser focusing axis to write a modification line. The glass plate was made of alkali silicate glass plate, of which basic properties are similar to those of sodalime glass. The thickness of the glass plate was $400 \mu \mathrm{m}$, and the focus of the laser pulses was about $375 \mu \mathrm{m}$ below the top surface.

After laser writing, the morphologies of the modification lines were observed by a transmission optical microscope, and the birefringence distributions originated from stresses in and around the modification lines were observed by a polarization microscope.

\subsection{Temporal energy modulation of laser pulses}

The energies of laser pulses were temporally modulated by inputting a voltage waveform (RF signal) to the laser system from an arbitrary waveform generator (SIGLENT, SDG5082) through a SMA cable. The voltage waveform modulated an acousto-optic modulator in the laser that controlled the output energies of laser pulses.

The temporal energy modulation in this study were three patterns; sinusoidal, ramp and square-patterns. The modulated pulse energies of three patterns were shown in Fig. 2(b). The frequency of the modulation was from $1 \mathrm{kHz}$ to $10 \mathrm{kHz}$. In this paper, we focused on the results with the modulation frequency of $1 \mathrm{kHz}$.

\section{Result}

We compared the morphologies of laser written modification lines, stress distributions and crack threshold with and without temporal energy modulation. We chose the modulation frequency from $1 \mathrm{kHz}$ to $10 \mathrm{kHz}$ based on the expectation that the temperature elevation inside the glass by heat accumulation occurs within $1 \mathrm{~ms}$ [1]. Because we found the similar results with energy modulation at $1 \mathrm{kHz}-10 \mathrm{kHz}$, we focused on the result obtained by the modulation frequency of $1 \mathrm{kHz}$ in this paper.

\subsection{Difference in modification lines with and without temporal modulation}

First, we compared the morphologies of ps laser written modification lines inside an alkali-silicate glass and stress distributions with and without temporal energy modulation. In this section, the modulation pattern was sinusoidal.

Figures 3 (a) and 3 (b) show transmission optical microscope images of modification lines written inside a glass by ps laser irradiation with and without temporal energy modulation, respectively. The average pulse energy 
was $10 \mu \mathrm{J}$, the repetition rate was $500 \mathrm{kHz}$ and the modulation frequency was $1 \mathrm{kHz}$. Clearly, the boundaries of the modification line without modulation were curved randomly (Fig. 3 (a)). On the other hand, the boundaries of the modification line were almost straight (Fig. 3 (b)), although a periodic pattern appeared in the central region of the line.

The stress distributions were much more different between modification lines with and without modulation. Figures 3 (c) and 3 (d) shows the birefringence images of the modification lines in the same areas of Fig. 3 (a) and 3 (b), respectively. Because birefringence inside a glass is originated from stress, the birefringence images reflect the stress distributions. In the modification line written without modulation (Fig. 3 (c)), the birefringence varied drastically inside the modification line. The drastic variation of the birefringence indicates that there was stress concentration inside the modification line. On the other hand, in the modification line written with modulation (Fig. 3 (d)), the birefringence was much smaller than that of Fig. 3 (c) and almost constant along the modification line. The smaller birefringence and the homogeneous distribution along the modification line means that the stress along the line was homogeneous and much smaller than that without modulation. The difference between the modification lines suggests that the stress concentration can be prevented by applying temporal energy modulation to ps laser irradiation.

There were also differences in the morphology between with and without modulation in the central part of the modification line. Figure 4 shows optical microscope images of the modification lines, of which focus was adjusted to make the structures in the central region clearly observed. In the central region of the modification line written without modulation (Fig. 4 (a)), the unclear structures were arrayed irregularly along the modification line. On the other hand, with modulation, ellipsoidal structures were arrayed periodically along the modification lines. This periodic structures may be responsible for formation of straight modification line with homogeneous stress distribution. The mechanism of the formation of straight modification line will be discussed in the section 4.1 in detail.

\subsection{Width and length of the modification lines}

To investigate the effect of the temporal energy modulation, we compared the width and the length of the modification lines and the threshold of crack generation with and without modulation. Here, we defined the "length" as the length of the modification line in the laser propagation direction. Figure 5 shows the plots of the widths and lengths of the modification lines as a function of average pulse energies. The variation of the width with pulse energy was almost the same with and without temporal energy modulation (Fig. 5(a)). However, the crack generation occurred at a lower pulse energy (about $16.5 \mu \mathrm{J}$ ) without modulation. On the other hand, the threshold of the crack generation with modulation was about $22 \mu \mathrm{J}$. As the result of higher threshold, the maximum width of the modification line became about 30 $\mu \mathrm{m}$ larger than that without modulation.
The length of the modification line varied with pulse

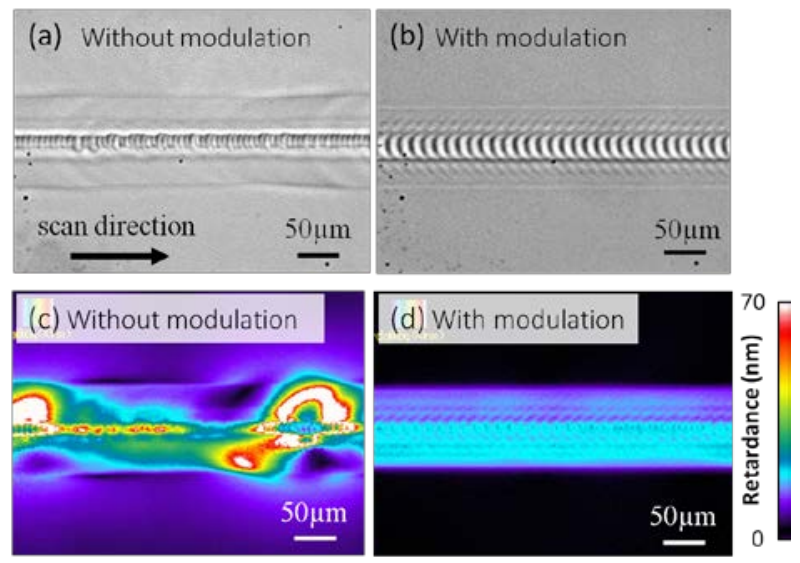

Fig. 3 (a), (b) Transmission optical microscope images of modification lines in alkali silicate glasses written by ps laser irradiation with and without temporal energy modulation (sinusoidal), respectiely. (c), (d) Birefringence images in the same areas of (a) and (b), respectively.

(a) Without modulation
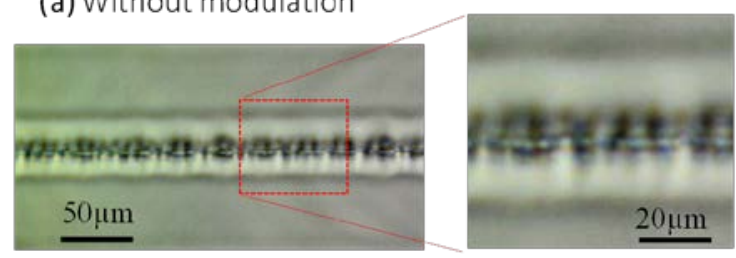

(b) With modulation

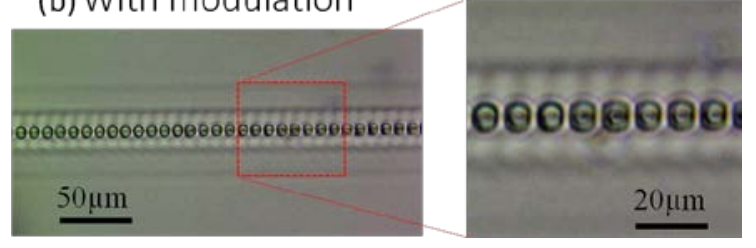

Fig. 4 Transmission optical microscope images of which focus was adjusted to make the structures in the central region of the modification line obaserved clearly. (a) Without energy modukation, and (b) with sinusoidal energy modulation.

energy differently with and without modulation (Fig. 5 (b)). The length of the modification line written without modulation increased about twice more steeply than that with modulation. Interestingly, the crack generation occurred when the length became about $270 \mu \mathrm{m}$ for both the cases.

\subsection{Influence of temporal modulation patterns}

In this section, we show different stress distributions in modification lines written by ps laser irradiation with temporal energy modulation of different patterns. Figure 6 shows retardance distributions of modification lines inside alkali-silicate glasses written with temporal energy modulation of three different patterns at the same average pulse energy $(10 \mu \mathrm{J})$. The modulation frequency was $1 \mathrm{kHz}$ for all the modulation patterns. The birefringence distributions show that stress was generated in the modification line and the stress along the modification line was homogeneous for all the modulation patterns. However, the stress amplitude and the width of the modification line 
depended on the modulation pattern. The stress amplitude and the line width were largest with the square-pattern modulation, and they were smallest with the ramp-pattern modulation.

(a)

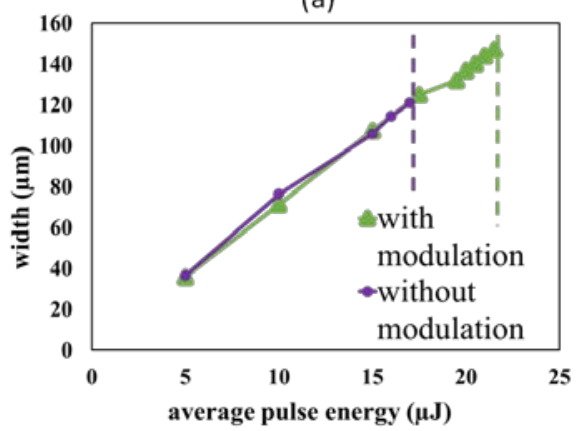

(b)

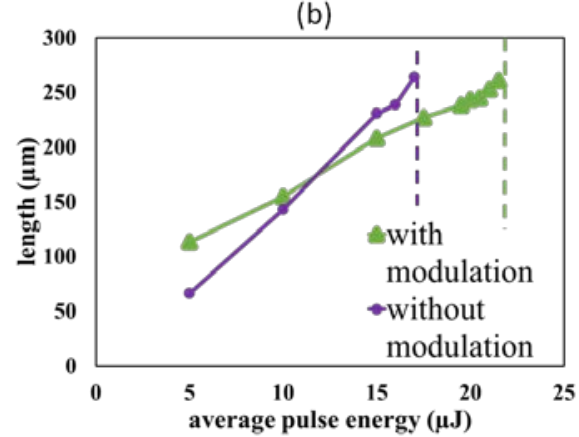

Fig. 5 Plots of the widths and lengths of modification lines written with and without temporal energy modulation against average pulse energies. The vertical broken lines indicate the threshold of crack generation.

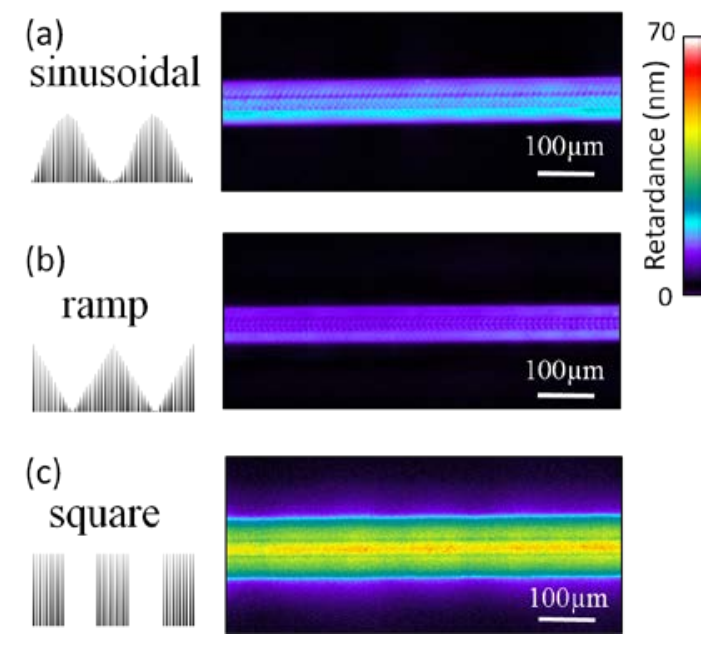

Fig. 6 Birefringence images in alkali-silicate glasses after writing modification lines by ps laser irradiation with three different temporal energy modulations.

Figure 7 shows the width and length of the modification lines plotted against the average pulse energy. These graphs indicate that the width and length of the modification lines were the most sensitive to the variation of the pulse energy with square-pattern modulation. The sensitivities to the pulse energy with sinusoidal and ramp-pattern modulation were similar, but the width and length of the modification line were larger with sinusoidal-pattern modulation at the same average energy.
Interestingly, the maximum width and length of the modification lines, which were measured near the threshold of crack generation, were almost the same with all the modulation patterns regardless of different

(a)

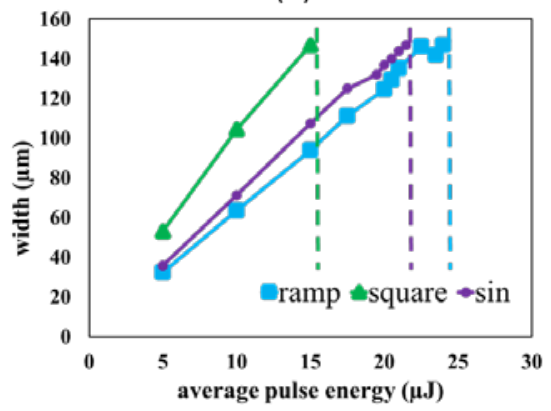

(b)

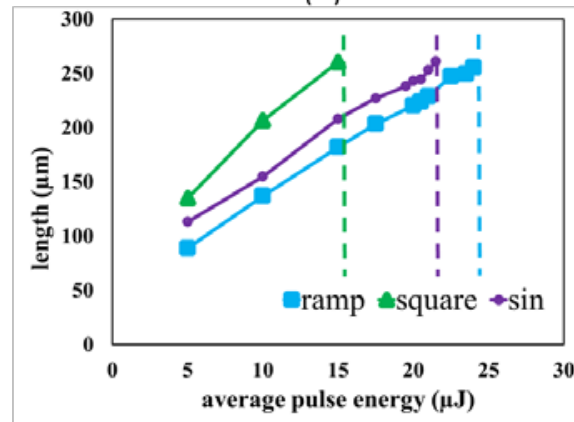

Fig. 7 Plots of the widths and lengths of the modification lines written with three different temporal energy modulations against average pulse energies. The vertical broken lines indicate the threshold of crack generation.

(a)
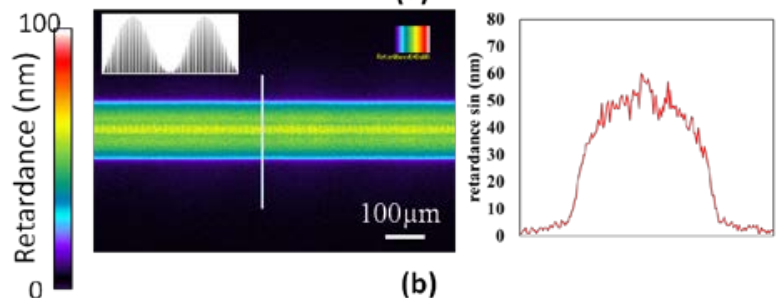

(b)

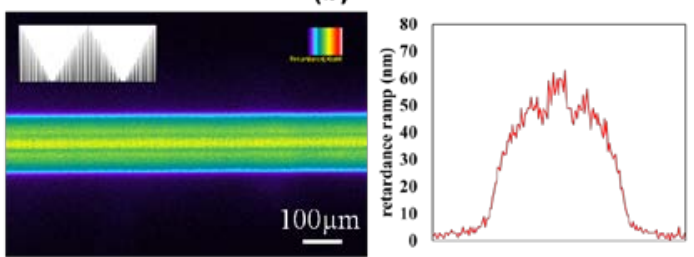

(c)
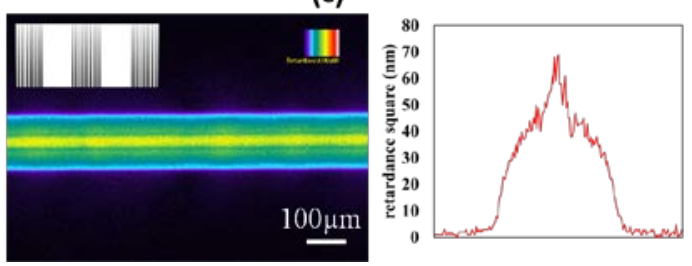

Fig. 8 Birefringence images of modification lines of the same width written with different temporal energy modulations. In the right of the images, the retardance profile across the modificaiton lines are plotted.

sensitivities to the pulse energy. The same maximum width and length of the modification line suggest that the 
threshold of the crack generation should be determined by the length of the modification line in the laser propagation direction and the relation between width and length does not depend on the modulation pattern.

Next, the birefringence distributions of the modification lines of the same width were compared to investigate the difference in the stress distributions with different modulation patterns. Figure 8 shows the retardance distributions of modification lines inside alkali-silicate glasses written with temporal modulation of three patterns. In all the cases, the retardances in the outer region of the modification lines were similar $(40-50 \mathrm{~nm})$, but the retardance at the center was different. To show the difference clearly, the retardance distributions across the modification lines were plotted in the right of the retardance images (Fig. 8). The distributions show that retardance across the modification line was uniform in the case of sinusoidal and ramp-patterns. On the other hand, there was a clear spike at the center of the modification line with square-pattern modulation. The difference suggests that sinusoidal and ramp-pattern modulation can suppress the generation of stress at the center of the modification line.

\section{Discussion}

\subsection{Mechanism of suppression of stress concentration with energy modulation}

We found that ps laser irradiation at $500 \mathrm{kHz}$ with temporal energy modulation enabled writing of modification lines with homogeneous and smaller stress generation. To consider the mechanism of stress generation in writing of modification lines with temporal energy modulation, we focus the difference in the central regions of the modification lines with and without modulation (Fig. 4). The microscope image of the modification line written without modulation shows that the irregularly shaped structures are arrayed along the modification line (Fig. 4 (a)). The irregular structures suggest that some irregularly occurring phenomena such as bubble formation and extremely large stress generation had occurred during writing of the modification line. On the other hand, ellipsoidal structures are arrayed periodically along the modification line written with modulation (Fig. 4 (b)). Because the distance between the periodic structures was determined by one cycle of the temporal energy modulation, we considered that the formation of periodic structures should be related to the mechanism of homogeneous and smaller stress generation.

Periodic structures in the modification lines written by high repetition rate ps laser irradiation have also been observed by several researchers. Nordin et al [15] observed the periodic movement of highly absorbing plasma during writing a modification line using a high-speed camera and attributed the periodical movement of plasma to the formation of periodic structures in a modification line (The periodic structures are different from those in Fig. 4(b).). Recently, observation of plasma emission by Nordin and numerical simulation by Miyamoto et al [14] elucidated the mechanism of periodic movement of highly light absorbing plasma. The observation and simulation showed that the plasma grows and moves in the laser propagation direction and suddenly decays at the position far from the laser focus. Before the decay of the plasma, new plasma is generated near the focus of the laser focus again.

The generation of highly absorbing plasma suggests that longer interaction time between plasma and laser field could induce higher temperature elevation as well as larger stress generation in the modified region inside a glass. In the case of laser irradiation at constant pulse energies (i. e. laser irradiation without temporal energy modulation), temperature elevation by increase of plasma would have occurred every cycle of the plasma movement. If the temperature elevation is excessive, bubble formation and strong stress generation could occur in the modified region, which result in irregular structures and stress concentration as shown in Fig. 3 (a), 3 (c) and 4 (a).

On the other hand, temporal energy modulation can suppress excess temperature elevation, because light energy absorbed by plasma can be controlled by changing pulse energies. The periodic structures in the modification line with modulation (Fig. 4 (b)) suggests that light energy absorbed by plasma and resulting temperature elevation should have increased and decreased periodically by modulating pulse energies. This periodic temperature change can be made smaller with temporal energy modulation by choosing an appropriate frequency of modulation. As the result, temporal energy modulation makes us to write modification lines with homogeneous and smaller stress (Fig. 3 (d)) and crack generation.

Another mechanism of stress generation with modulation is that temporal energy modulation could shorten the length of the plasma movement. This mechanism is supported by Figure 5(b), which shows that the length of the modification line increased with pulse energy more sensitively without modulation. It is expected that longer plasma movement could result in larger temperature change in the modified region because the light-plasma interaction time is longer. In addition, longer plasma movement induce larger temperature difference along the laser propagation direction. Because larger temperature change and larger temperature difference generate larger stress, the suppression of the plasma movement by temporal energy modulation can suppress the stress generation and crack formation.

Although the stress distribution became homogeneous by temporal energy modulation, the generation of ellipsoidal structures were observed in the central part of the modulation line (Fig. 4 (b)). We expect that these structures could not affect glass laser welding regardless of their void-like shapes, because they are not voids but just refractive index distribution. For this point, we are investigating the influences of the ellipsoidal structures on laser welding and how to suppress these structures by choosing appropriate energy modulation curves.

\subsection{Mechanism of different stress distribution by modulation pattern}

In the section 3.3, we described the influence of the modulation pattern on the stress distribution in modification lines. The first difference by modulation patterns is the width and length of modification lines at the same average pulse energy. Figure 6 and 7 show that larger modification lines can be written by laser irradiation with 
square-pattern modulation. This difference suggests that laser irradiation with constant pulse energies should be effective to enhance light absorption by plasma.

The other difference is the stress distributions in modification lines. The stress distributions in modification lines of the same width were compared in Fig. 8. It was found that there was a clear spike of stress in the modification line written with square-pattern modulation (Fig. 8 (c)), while the stress distributions were more uniform with the other modulation patterns (Fig. 8 (a) and 8 (b)). The spike of stress with square-pattern modulation suggests that sudden rise and fall of pulse energy during laser irradiation with this modulation could have induced sudden burst of larger stress in the modified region. On the other hand, the stress generation could be moderate with the other modulation patterns, because pulse energies change gradually with these modulation patterns. Because the stress concentration can be an origin of crack generation in glasses, sinusoidal and ramp-patterned modulations should be suitable for glass welding application.

\section{Conclusion}

In conclusion, stress and crack generation in writing of modification lines using ps laser were able to be suppressed by applying temporal modulation of pulse energies. In addition, it was found that temporal modulations of sinusoidal and ramp patterns are suitable for writing modification lines with less stress concentration. Based on the mechanism of light absorption by plasma during laser irradiation at a high repetition rate, we interpreted that drastic temperature change had been suppressed by changing pulse energies during laser irradiation, and the suppression contributed to stable laser writing of modification lines with less stress and crack generation. This technique has a great impact to improve the breaking strength of glasses after laser welding.

\section{Acknowledgments}

This work was supported by the Cross-Ministerial Strategic Innovation Promotion (SIP) Program.

\section{Reference}

[1] C. B. Schaffer, J. F. Garcia, E. Mazur: Appl. Phys. A 76 (2003) 351.

[2] S. M. Eaton, H. Zhang, P. R. Herman, F. Yoshino, L. Shah, J. Bovatsek, A. Y. Arai: Opt. Express, 13, (2005) 4708.

[3] M. Shimizu, M. Sakakura, M. Ohnishi, Y. Shimotsuma, T. Nakata, K. Miura, K. Hirao: J. Appl. Phys., 108, (2010) 073533.

[4] I. Miyamoto, K. Cvecek, Y. Okamoto, M. Schmidt: Appl. Phys. A, 114 (2014) 187.

[5] T. Tamaki, W. Watanabe, J. Nishii: Jpn. J. Appl. Phys., 44, (2005) L687.

[6] I. Miyamoto, A. Horn, J. Gottmann: J. Laser Micro/Nanoeng., 2, (2008) 7.

[7] S. Richter, S. Döring, A. Tünnermann, S. Nolte: Appl. Phys. A 103, (2011) 257.

[8] R. M. Carter, J. Chen, J. D. Shephard, R. R. Thomson, D. P. Hand: Appl. Opt., 53, (2014) 4233.

[9] C. B. Schaffer, A. Brodeur, E. Mazur: Meas. Sci. Tech., 12, (2001) 1784.

[10] R. Knechtel: Microsyst. Technol., 12, (2005) 63.

[11] M. Sun, U. Eppelt, W. Schulz, J. Zhu: Opt. Mater Express, 3, (2013) 1716.

[12] B. C. Stuart, M. D. Feit, A. M. Rubenchik, B. W. Shore and M. D. Perry: Phys. Rev. Lett., 74, (1995) 2248.

[13] A. Vogel, J. Noack, G. Huttman and G. Paltauf: Appl Phys. B, 81, (2005) 1015.

[14] I. Miyamoto, Y. Okamoto, R. Tanabe, Y. Ito, K. Cvecek, M. Schmidt: Opt. Express, 24, (2016) 25718.

[15] I. H. W. Nordin, Y. Okamoto, A. Okada, H. Jiang, T. Sakagawa: Appl. Phys. A, 122, (2016) 492.

(Received: June 21, 2017, Accepted: August 16, 2017) 